\title{
TOWARDS IMPROVED CIRRUS CLOUD OPTICAL DEPTHS FROM CALIPSO
}

\author{
Anne Garnier ${ }^{1,2 *}$, Mark Vaughan ${ }^{2}$, Jacques Pelon $^{3}$, David Winker ${ }^{2}$, Chip Trepte ${ }^{2}$, and Stuart \\ Young $^{4}$ \\ ${ }^{I}$ Science Systems and Applications, Inc., Hampton, VA, 23666,USA, *Email: anne.garnier@latmos.ipsl.fr \\ ${ }^{2}$ NASA Langley Research Center, Hampton, VA, 23681, USA \\ ${ }^{3}$ Université Pierre et Marie Curie, CNRS, IPSL, LATMOS, Paris, 75252, FRANCE \\ ${ }^{4}$ CSIRO Oceans \& Atmosphere, Aspendale, Victoria, 3195, AUSTRALIA
}

\begin{abstract}
This paper reviews recent advances regarding the retrieval of optical depths of semi-transparent cirrus clouds using synergetic analyses of perfectly collocated observations from the CALIOP lidar and the IIR infrared radiometer aboard the CALIPSO satellite.
\end{abstract}

\section{INTRODUCTION}

The CALIPSO satellite, launched nearly 9 years ago, carries the CALIOP (Cloud and Aerosol Lidar with Orthogonal Polarization) lidar operating at two wavelengths (532 and $1064 \mathrm{~nm}$ ), the 3-channel Imaging Infrared Radiometer (IIR) operating in the 8-12 $\mu \mathrm{m}$ thermal infrared spectral range, and the Wide Field-of-view Camera (WFC) operating in the visible domain [1]. Observations from the three instruments are almost perfectly temporally and spatially collocated along the lidar track, allowing detailed synergetic analyses. In particular, visible $(532 \mathrm{~nm})$ extinction optical depths retrieved from CALIOP [2] have been extensively compared with absorption optical depths retrieved at $12.05 \mu \mathrm{m}$ from the IIR [3]. Indeed, CALIOP and IIR retrieval techniques are based on independent physical principles, and are subject to different sources of uncertainty. To ensure meaningful comparisons between the active and passive observations, this study examines semi-transparent clouds that do not fully attenuate the CALIOP beam. In addition, only atmospheric columns containing one single cloud layer are considered. At night, CALIOP retrievals use one of two different techniques, which are briefly reviewed in section 2, and the consistency between the three independent retrievals is initially investigated in section 3 . The CALIOP multiple scattering correction factor inferred from refined comparisons between CALIOP and IIR is then presented in section 4, which is followed by our conclusions in section 5 .

\section{CALIOP CIRRUS OPTICAL DEPTHS}

Visible optical depths, $\tau_{\text {vis, }}$, for semi-transparent cirrus measured by CALIOP are retrieved using one of two totally independent techniques. The first consists in directly measuring the layer apparent 2-way transmittance, $\mathrm{T}_{\mathrm{c}}{ }^{2}$, on those occasions when clear air is found immediately above and below the layer [2]. For these layers, $\mathrm{T}_{\mathrm{c}}{ }^{2}$ is obtained directly from the ratio of the mean attenuated scattering ratios in clear air regions above and below the cloud, and hence is insensitive to calibration errors. This technique allows a direct determination of the layer "apparent" optical depth, $\eta \cdot \tau_{\text {vis }}$, where $\eta$ represents the required correction for the contribution from multiple scattering. Thus, $\tau_{\mathrm{vis}}$ can be derived as:

$$
\tau_{\mathrm{vis}}=\frac{-\ln \left(\mathrm{T}_{\mathrm{c}}^{2}\right)}{2 \eta}
$$

In the version 3 algorithm, $\eta$ is assumed constant throughout the layer, identical in-cloud and below cloud, and equal to 0.6. The lidar ratio, $\mathrm{S}_{\mathrm{c}}$, can then be retrieved from the particulate layerintegrated attenuated backscatter, $\gamma^{\prime}$, as [4]:

$$
S_{c}=\frac{1-e^{-2 \eta \tau_{v i s}}}{2 \eta \gamma^{\prime}}
$$

Note that in practice, this initial estimate of $\mathrm{S}_{\mathrm{C}}$ is refined iteratively until the integral of the retrieved extinction profile exactly matches the 
directly-determined optical depth [2]. In the version 3 algorithm, this so-called "constrained retrieval' is applied when the uncertainty in $S_{c}$ is estimated to be less than $40 \%$. This condition, which requires relatively large signal-to-noise ratios in the clear air regions, is fulfilled almost exclusively by nighttime measurements. Furthermore, because the signal is noisy, the measured optical depth distribution is increasingly truncated as true optical depth decreases, because a larger fraction of these small optical depths may become negative or do not satisfy the estimated relative uncertainty requirement [5]. Consequently, both the optical depths and the lidar ratios obtained from constrained retrievals exhibit a high bias at optical depths smaller than about 0.6 that increases as optical depth decreases [6]. For consistency, optical depths reported in the CALIOP $5-\mathrm{km}$ cloud layer product are derived from the measured apparent 2-way transmittance (Eq. 1) only when the conditions imposed by the constrained technique are satisfied.

When the constrained technique is deemed not possible, $\tau_{\text {vis }}$ reported in the products is retrieved using an initial lidar ratio which is set to $25 \mathrm{sr}$ in version 3 data products. Within the retrieval code, this initial lidar ratio can be increased or reduced to prevent extinction retrievals from diverging [2]. For semi-transparent cirrus clouds, most of the retrievals are 'unconstrained', with final lidar ratios identical to the initial ones. Nevertheless, the apparent 2-way transmittance derived for those layers is also reported in the products, from which the layer optical depth can be derived using Eq. 1, and compared with optical depths retrieved using $\mathrm{S}_{\mathrm{c}}=25 \mathrm{sr}$.

\section{COMPARISONS WITH IIR CIRRUS OPTICAL DEPTHS}

The IIR optical depth retrieved at $12.05 \mu \mathrm{m}, \tau_{\mathrm{a}}$, is essentially an absorption optical depth derived from the cloud emissivity, $\varepsilon$, as [3]:

$$
\tau_{a}=-\ln (1-\varepsilon)
$$

where

$$
\varepsilon=\frac{R_{m}-R_{B G}}{R_{B B}-R_{B G}}
$$

In Eq. $4, R_{m}$ is the measured radiance. $R_{B B}$ is the radiance at the top of the atmosphere (TOA) of a blackbody source located at the centroid altitude of the attenuated backscatter profile whose thermodynamic temperature, $T_{c}$, is retrieved from ancillary meteorological data. $R_{B G}$ represents the correction for the TOA background radiance which would be observed had the cloud not been present. $R_{B G}$ is preferably constrained by observations in neighboring pixels. Otherwise, $R_{B G}$ is computed in real-time using the FASRAD radiative transfer model [7] and ancillary atmospheric and surface data.

Fig. 1 shows $2 \mathrm{D}$-histograms of nighttime $\tau_{\mathrm{vis}}$ and $2 \tau_{\mathrm{a}}$ for single-layered semi-transparent cirrus. All clouds were measured over oceans and classified (with high confidence) as being composed of randomly oriented ice (ROI). In order to minimize possible biases, IIR $\tau_{\mathrm{a}}$ is taken from $\mathrm{R}_{\mathrm{BG}}$ measured in neighboring pixels. The $\tau_{\text {vis }} / \tau_{\mathrm{a}}$ ratio is relatively insensitive to cirrus cloud microphysical properties. For this data set, it is expected to be mostly around 2 [6]. In Fig.1a, CALIOP $\tau_{\text {vis }}$ is as reported in the version 3 products. Two ensembles of samples are clearly seen. The larger values of $\tau_{\text {vis }}$ are from the constrained retrievals and hence the 2-way transmittance (Eq. 1), whereas the smaller values of $\tau_{\mathrm{vis}}$ are from unconstrained retrievals and the default lidar ratio $S_{c}=25 \mathrm{sr}$. The clear discontinuity between both ensembles in terms of comparison with IIR is explained by the fact that the default lidar ratio is not consistent with the lidar ratios derived from the constrained retrievals, and is too small by about $25 \%$, as discussed in [8]. In Fig. $1 \mathrm{~b}, \tau_{\text {vis }}$ of clouds for which unconstrained retrievals are chosen in the version 3 algorithm is instead obtained from the apparent 2-way transmittance (Eq. 1). Here, no discontinuity between the two ensembles of retrievals is seen, which confirms that the default lidar ratio is too small in the mean for the unconstrained retrievals. Thus, it appears that the constrained technique could be extended to smaller optical depths, and likely to more daytime measurements, by relaxing the threshold in the relative lidar ratio uncertainties used in the version 3 algorithm, which, in turn, would substantially decrease the number of unconstrained retrievals. Analyses in [6] indicate that constrained retrievals could thus be extended down to $2 \tau_{\mathrm{a}}=0.3$ with no evidence of systematic high bias. It is noted that below this limit, the 
number of non-physical negative values of $\tau_{\text {vis }}$ increases rapidly due to the unavoidable increasing noise.
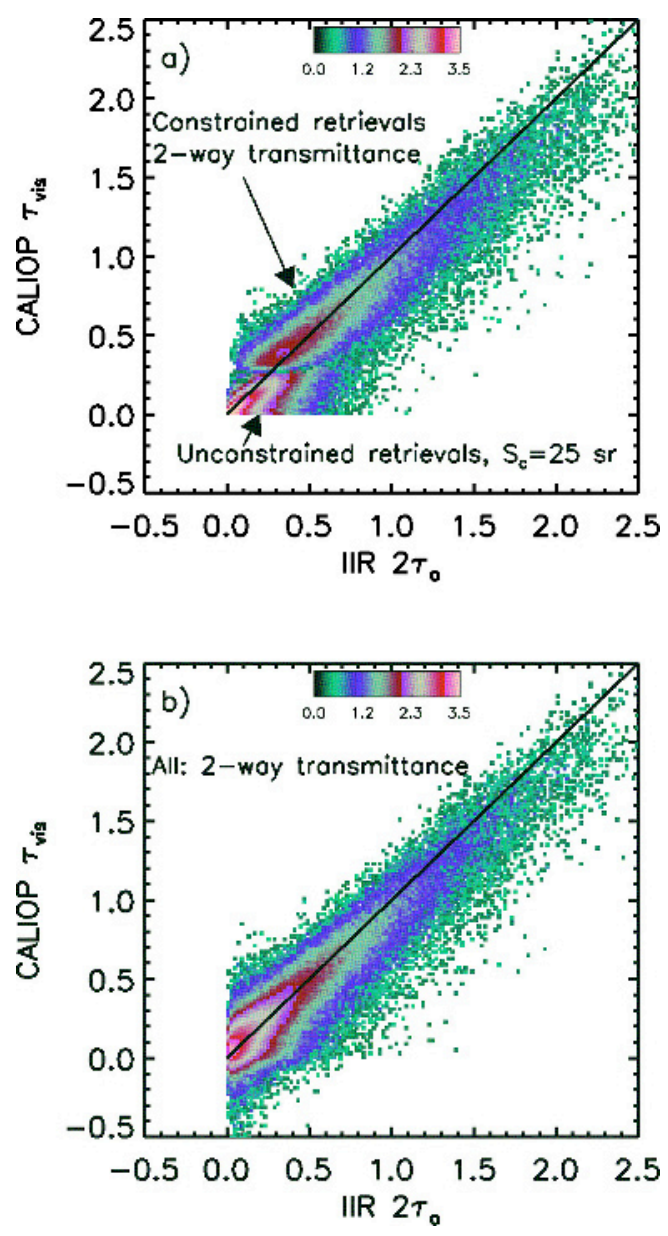

Fig. 1. 2D-histogram of nighttime CALIOP visible optical depth $\left(\tau_{\text {vis }}\right)$ and 2 XIIR absorption optical depth at $12.05 \mu \mathrm{m}\left(2 \tau_{\mathrm{a}}\right)$ with $\mathrm{R}_{\mathrm{BG}}$ constrained by neighboring observations for single-layered semi-transparent cirrus composed of ROI (high confidence) over oceans during 2008. a) CALIOP version 3 products; b) for unconstrained retrievals, $\tau_{\mathrm{vis}}$ is from the apparent 2-way transmittance and not from $\mathrm{S}_{\mathrm{c}}=25 \mathrm{sr}$. The black line represents $\tau_{\text {vis }} / \tau_{\mathrm{a}}=2$. The color code is the decimal logarithm of the number of samples.

\section{MULTIPLE SCATTERING FACTOR}

Fig.1b shows that the relationship between the CALIOP $\tau_{\text {vis }}$ derived from the apparent 2-way transmittance and the IIR $\tau_{\mathrm{a}}$ is overall close to the expectations. However, detailed analyses show that the $\tau_{\text {vis }} / \tau_{\mathrm{a}}$ ratio increases as the temperature $\mathrm{T}_{\mathrm{c}}$ decreases, which is in disagreement with theo- retical simulations and microphysical properties derived from the IIR microphysical algorithm [6]. As the CALIOP multiple scattering correction factor $\eta$ is the only free parameter in these analyses, the proposed explanation is a temperaturedependent multiple scattering factor, $\eta_{\mathrm{T}}$. Fig. 2 shows $\eta_{T}$ against $T_{c}$ inferred from these comparisons [6] by following the approach initially introduced in [4].

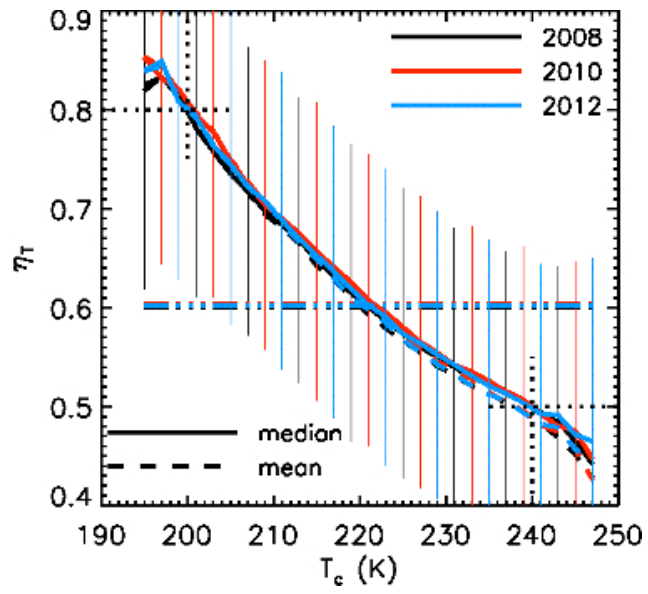

Fig. 2: CALIOP median (solid) and mean (dashed) multiple scattering factor \pm standard deviation inferred from the ratios of CALIOP visible optical depth $\tau_{\text {vis }}$ derived from the apparent 2-way transmittance to IIR absorption optical depth at $12.05 \mu \mathrm{m}\left(\tau_{\mathrm{a}}\right)$. The horizontal dashed dotted lines are the mean values overall. See text for details.

Clouds are composed of ROI (high confidence), and those clouds with base temperatures warmer than $-20^{\circ} \mathrm{C}$ are discarded to minimize possible contamination from mixed phase clouds. IIR background references are from both measurements in neighboring pixels and computations to maximize the number of samples. Analyses are over oceans, and $2 \tau_{\mathrm{a}}$ is limited to values larger than 0.3 to minimize residual biases at small optical depth for both CALIOP and IIR. $\tau_{a}$ is corrected for biases in $R_{B B}$ that have been quantified using CALIOP extinction profiles [6], and is on average increased by $1 \%$ at $2 \tau_{\mathrm{a}}=0.3$ up to $7 \%$ at $2 \tau_{\mathrm{a}}=2$. The median (solid lines) and mean (dashed lines) values of $\eta_{\mathrm{T}}$ are shown for 2008 (black), 2010 (red), and 2012 (blue), demonstrating a remarkable repeatability from 
one year to the other. The median $\eta_{\mathrm{T}}$ is found to decrease from $\eta_{\mathrm{T}}=0.8$ at $200 \mathrm{~K}$ to $\eta_{\mathrm{T}}=0.5$ at 240 $\mathrm{K}$. The horizontal dashed dotted lines show the mean values overall, which are $0.601,0.604$, and 0.602 respectively in 2008,2010 , and 2012 . These values are found to be essentially identical to the constant value $\eta=0.6$ taken in the version 3 operational algorithm. $\mathrm{S}_{\mathrm{c}}$ is found to be weakly varying with $T_{c}$ when $\eta$ is taken constant. When using $\eta_{\mathrm{T}}$ from Fig. 2, $S_{\mathrm{c}}$ is found to increase by $50 \%$ from $\mathrm{T}_{\mathrm{c}}=200 \mathrm{~K}$ to $\mathrm{T}_{\mathrm{c}}=228 \mathrm{~K}$ and to be mostly constant at warmer temperatures.

\section{CONCLUSIONS}

Previous analyses showed that CALIOP constrained and unconstrained retrieval techniques are in good agreement when a lidar ratio derived from the statistical analysis of constrained retrievals is chosen as a default lidar ratio for CALIOP unconstrained retrievals. Here, we report that constrained retrievals can be improved, in the mean, by eliminating the threshold on the estimated relative lidar ratio uncertainties used in the version 3 algorithm. At the same time, this significantly increases the number of optical depths obtained directly from measurements rather than by initializing the retrievals with a default lidar ratio. Furthermore, analyses of the relationship between visible extinction optical depths derived from the apparent 2-way transmittances and infrared absorption optical depths indicate that the CALIOP multiple scattering correction factor, taken equal to 0.6 in the version 3 algorithm, varies from 0.8 at $200 \mathrm{~K}$ to 0.5 at 240 $\mathrm{K}$. The mean value overall is found essentially equal to 0.6 , so that on average, CALIOP visible optical depths compare as expected with IIR effective optical depths at $12.05 \mu \mathrm{m}$. These findings will be used as guidance for the definition of the future version 4 of the products.

\section{ACKNOWLEDGEMENTS}

The authors are thankful to the Data Management Team at NASA/LaRC and to ICARE data center in Lille. CNES is acknowledged for its support.

\section{REFERENCES}

[1] Winker, D. M., J. Pelon, J. A. Coakley, Jr., S. A. Ackerman, R. J. Charlson, P. R. Colarco, P.
Flamant, Q. Fu, R. Hoff, C. Kittaka, T. L. Kubar, H. LeTreut, M. P. McCormick, G. Megie, L. Poole, K. Powell, C. Trepte, M. A. Vaughan, B. A. Wielicki, 2010: The CALIPSO Mission: A global 3D view of aerosols and clouds, Bull. Am. Meteorol. Soc., 91, 1211-1229.

[2] Young, S. A. and M. A. Vaughan, 2009: The retrieval of profiles of particulate extinction from Cloud Aerosol Lidar Infrared Pathfinder Satellite Observations (CALIPSO) data: Algorithm description, J. Atmos. Oceanic Technol., 26, 1105-1119.

[3] Garnier, A., J. Pelon, P. Dubuisson, M. Faivre, O. Chomette, N. Pascal, D. P. Kratz, 2012: Retrieval of cloud properties using CALIPSO Imaging Infrared Radiometer. Part I: effective emissivity and optical depth, J. Appl. Meteor. Climatol., 51, 1407-1425.

[4] Platt, C. M. R., 1973: Lidar and radiometer observations of cirrus clouds, J. Atmos. Sci., 30, 1191-1204.

[5] Young, S. A., M. A. Vaughan, R. E. Kuehn, D. M. Winker, 2013: The retrieval of profiles of particulate extinction from Cloud-Aerosol Lidar Infrared Pathfinder Satellite Observations (CALIPSO) data: uncertainty and error sensitivity analyses, J. Atmos. Oceanic Technol., 30, 395428.

[6] Garnier, A., J. Pelon, M. A. Vaughan, D. M. Winker, C. R. Trepte, P. Dubuisson, 2015: Optical depths of semi-transparent cirrus clouds over oceans from CALIPSO infrared radiometer and lidar measurements, and an evaluation of the lidar multiple scattering factor, Atmos. Meas. Tech. Discuss., 8, 2143-2189.

[7] Dubuisson P., V. Giraud, O. Chomette, H. Chepfer, J. Pelon, 2005: Fast radiative transfer modeling for infrared imaging radiometry, $J$. Quant. Spectr. Rad. Tr., 95, Issue 2, 1 October 2005, 201-220.

[8] Garnier, A., M. A. Vaughan, P. Dubuisson, D. Josset, J. Pelon, D. M. Winker, 2012: Multi-sensor cirrus optical depth estimates from CALIPSO, in: Reviewed \& Revised Papers Presented at the 26th International Laser Radar Conference, Porto Heli, Greece, 25-29 June 2012, 691-694. 